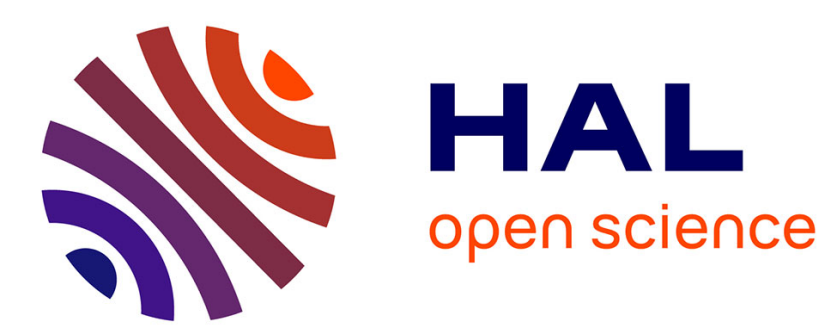

\title{
Un algorithme capable de prédire les décisions des juges : vers une robotisation de la justice?
}

Boris Barraud

\section{To cite this version:}

Boris Barraud. Un algorithme capable de prédire les décisions des juges: vers une robotisation de la justice?. Les Cahiers de la justice, 2017, 1, pp.121-139. hal-01404518v2

\section{HAL Id: hal-01404518 \\ https://hal.science/hal-01404518v2}

Submitted on 11 Sep 2017

HAL is a multi-disciplinary open access archive for the deposit and dissemination of scientific research documents, whether they are published or not. The documents may come from teaching and research institutions in France or abroad, or from public or private research centers.
L'archive ouverte pluridisciplinaire HAL, est destinée au dépôt et à la diffusion de documents scientifiques de niveau recherche, publiés ou non, émanant des établissements d'enseignement et de recherche français ou étrangers, des laboratoires publics ou privés. 


\title{
Un algorithme capable de prédire les décisions des juges : vers une robotisation de la justice?
}

\author{
Boris Barraud \\ Docteur en droit, Laboratoire interdisciplinaire de droit des médias et des mutations sociales \\ (EA n 4328), Université d'Aix-Marseille
}

\begin{abstract}
Les robots s'immiscent chaque jour un peu plus dans les activités humaines et sociales, à tel point que, lors de la dernière élection présidentielle américaine, l'un des candidats déclarés n'était autre que Watson, le superordinateur de la firme IBM. En matière judiciaire, les derniers progrès des "legaltechs » invitent à prendre au sérieux le sujet de la robotisation de la justice. En ce sens, des chercheurs britanniques et américains ont publié, le 24 octobre 2016, les résultats de travaux visant à créer une intelligence artificielle capable de deviner les décisions de justice. Confronté à près de 600 affaires jugées par la Cour européenne des droits de l'homme, leur algorithme a retenu huit fois sur dix la même solution que les magistrats. Le fait qu'un juge-robot opère le plus souvent les mêmes choix que des juges-humains accrédite la vision d'une justice fonctionnant à base de syllogisme, contre la thèse d'une justice abandonnée à la subjectivité de chaque magistrat. Par ailleurs, cette expérience de justice algorithmique pose les questions de la possibilité et de l'opportunité d'une future justice rendue par ordinateur ou, du moins, assistée par ordinateur. Enfin, les services dits de "justice prédictive », qui se développent aux États-Unis mais aussi en France autour de savants algorithmes, peuvent également constituer des aides à la défense et au conseil juridique en permettant à tout justiciable de mesurer la probabilité de remporter un éventuel procès.
\end{abstract}

British and American researchers have just created an artificial intelligence capable of predicting court decisions. In almost 600 cases judged by the European Court of Human Rights, the algorithm has adopted the same solution as judges eight times out of ten. The fact that a judge-robot most often makes the same choices as human judges credited the thesis of a syllogistic and mechanical justice, not the thesis of a justice left to the subjectivity of the judges. Moreover, this algorithmic justice experience raises the question of a computer-assisted justice. Finally, predictive justice, which is developing particularly in the United States, can also help lawyers by allowing them to estimate the probability of winning a case.

«Les prophéties relatives aux décisions qu'en fait prendront les cours et tribunaux, et rien de plus » 1 , telle est la conception du droit promue par Oliver Wendell Holmes, illustre juge à la Cour suprême des États-Unis et pionner du courant de théorie du droit dit « réaliste » - lequel invite à concentrer toute l'attention sur les activités des tribunaux. Certainement savoir ce que les juges feront de leurs cas personnels et non savoir ce que les lois prévoient en termes généraux et impersonnels est-il ce qui intéresse en premier lieu les justiciables. Aussi différents chercheurs ont-ils déjà réfléchi à la possibilité de créer des outils permettant, en croisant les faits et le droit pertinent, d'anticiper les issues des procès. En 1963, l'avocat

${ }^{1}$ O. W. Holmes, La voie du droit (1897), trad. L. De Sutter, Dalloz, coll. Tiré à part, 2014, p. 9. 
Reed Lawlor avait jeté les bases d'un traitement informatique des données d'un dossier afin d'anticiper sa recevabilité ou irrecevabilité ${ }^{1}$. Il estimait qu'une compréhension des méthodes d'interprétation des faits et du droit par les juges pourrait permettre de dégager des constances et de réaliser des prophéties scientifiques des décisions qu'en fait prendront les cours et tribunaux. D'autres, au cours du $\mathrm{XX}^{\mathrm{e}} \mathrm{s}$., se sont essayés à la construction de modèles mathématiques $^{2}$ ou reposant sur des probabilités ${ }^{3}$ ou des corrélations ${ }^{4}$ censés permettre d'anticiper, avec plus ou moins de précision, les jugements et arrêts ${ }^{5}$. Récemment, le chercheur américain Josh Blackman a mis au point un modèle mathématique grâce auquel les verdicts de la Cour suprême des États-Unis peuvent être déterminés avec un taux de fiabilité atteignant $75 \%^{6}$.

Les récents progrès technologiques, notamment dans les domaines du traitement du langage naturel et de l'apprentissage automatique, ont permis de franchir une nouvelle étape en matière de justice «prédictive», «prévisible», «quantitative», «statistique » ou «simulative ${ }^{7}$. Ainsi une équipe de chercheurs en informatique, en psychologie positive, en science de l'information et en droit $^{8}$ a-t-elle élaboré un algorithme capable de deviner les décisions de justice en croisant les faits, les arguments des parties et le droit positif pertinent. Les scientifiques ont publié les résultats de leurs recherches le 24 octobre 2016, dans un article intitulé «Predicting Judicial Decisions of the European Court of Human Rights: a Natural Language Processing Perspective $»$.

Capable d'opérer les mêmes choix que les juges-humains dans huit cas sur dix, cet algorithme ne manque pas de réveiller les plus sombres inquiétudes des techno-pessimistes liées à la robotisation des sociétés, l'imagination amenant à anticiper un avenir dans lequel les malfaiteurs seraient arrêtés par des robots-policiers et les litiges tranchés par des robots-juges. Sous un angle plus rationnel, il pose la question de l'évolution des professions judicaires vers un remplacement des hommes par des machines «intelligentes» disant le droit automatiquement ou, plus sûrement, vers une association des hommes et de ces machines

${ }^{1}$ R. C. LAWLOR, «What Computers Can Do: Analysis and Prediction of Judicial Decisions », American Bar Association Journal 1963, p. 337 s.

${ }^{2}$ R. KEOWN, « Mathematical Models for Legal Prediction », Computer/LJ 1980, p. 829 s.

${ }^{3}$ J. A. SEgAL, « Predicting Supreme Court Cases Probabilistically: the Search and Seizure Cases (1962-1981)», American Political Science Review 1984, p. 891 s.

${ }^{4}$ S. S. NAGEL, « Applying correlation analysis to case prediction », Texas Law Review 1963, p. $1006 \mathrm{~s}$.

${ }^{5}$ F. KORT, «Predicting Supreme Court Decisions Mathematically: a Quantitative Analysis of the "Right to Counsel" Cases », American Political Science Review 1957, $\mathrm{n}^{\circ}$ 51, p. 1 s.

${ }^{6}$ A. Aft, J. Blackman, C. M. CARPEnter, «FantasySCOTUS: Crowdsourcing a Prediction Market for the Supreme Court», Northwestern Journal of Technology \& Intellectual Property 2012, $\mathrm{n}^{\circ} 10$, p. 125 s.; J. Blackman, M. J. Bommarito, D. M. KATZ, «Predicting the Behavior of the Supreme Court of the United States: A General Approach », SSRN Electronic Journal 21 juill. 2014 (disponible à 1'adresse <ssrn.com/abstract=2463244>).

${ }^{7} \mathrm{Si}$ l'on a pris l'habitude d'utiliser l'expression «justice prédictive », d'aucuns préfèrent recourir à des qualificatifs concurrents car il ne s'agit pas à proprement parler de prédiction mais plutôt de calcul, de logique et de statistique.

${ }^{8}$ Ces chercheurs proviennent de l'Université de Londres, de l'Université de Sheffield et de l'Université de Pennsylvanie.

${ }^{9}$ N. Aletras, V. Lampos, D. Tsarapatsanis, D. PreoțiuC-Pietro, « Predicting Judicial Decisions of the European Court of Human Rights: a Natural Language Processing Perspective », Peer Journal of Computer Science 24 oct. 2016 (disponible à l'adresse <peerj.com/articles/cs-93/\#aff-1>). 
« intelligentes », laquelle pourrait permettre des gains de temps et des économies de moyens considérables. À l'heure où la précarisation de la justice est régulièrement dénoncée, ne faut-il pas voir dans cet algorithme une technologie prometteuse?

D'un point de vue théorique, le fait qu'un robot soit en mesure de rendre la même justice qu'un humain dans huit cas sur dix, mais aussi le fait que les décisions du juge-robot et du juge-humain divergent deux fois sur dix, sont potentiellement riches d'enseignements. L'illustre théoricien du droit italien Norberto Bobbio observait que, «depuis l'époque de ce qu'on a nommé le fétichisme de la loi, beaucoup d'eau est passée sous les ponts et personne ne croit plus sérieusement au juge automate ${ }^{1}$. Néanmoins, qu'un juge-humain et un jugerobot, en présence de mêmes faits, aboutissent très souvent à la même solution, n'est-ce pas le signe d'une justice quasi-machinale ordonnée autour du syllogisme - ce dont il faudrait se féliciter sous l'angle de la sécurité juridique et de la prévisibilité de la justice ?

En particulier, l'expérience de justice algorithmique interroge le courant théorique dit « réaliste ». Celui-ci se concentre sur le droit en action plutôt que sur le droit des textes, droit en action qui se laisse surtout observer au sein des prétoires. Si le droit se définit en tant que « décision du souverain ${ }^{2}$, ce souverain, pour les tenants du réalisme juridique, serait moins le parlement-législateur que le juge. Selon les réalistes, les lois ne feraient pas partie du droit positif ; au mieux, elles seraient une source matérielle, soit une source d'inspiration, et seuls les jugements constitueraient le droit positif. Ils bannissent, quels que soient le contenu de la loi et les données de l'espèce, le recours au raisonnement de type déductif, syllogistique et linéaire.

Ensuite, peut-être le cadre d'analyse le plus «réaliste », donc le plus conforme aux enseignements de l'expérience, réside-t-il dans la version scandinave du réalisme — par opposition à sa version américaine ${ }^{3}$, plus radicale. Le réalisme scandinave retient, entre autres propositions, que, si les juges sont de fait les véritables créateurs des normes, ils n'en sont pas moins fortement influencés par les lois et règlements, si bien qu'en présence de faits $a$, un juge-humain $x$, un autre juge-humain $y$ et un juge-robot $z$ en viendront logiquement à prononcer peu ou prou la même décision.

Si les tribunaux, bien que libres car nul ne juge les juges, rendent leurs verdicts en appliquant syllogistiquement le droit aux faits, peut-être le font-ils souvent mais non systématiquement. En effet, pour ce qui est des deux cas sur dix dans lesquels les décisions du juge-humain et du juge-robot divergent, il n'est pas impossible que les sentences découlant le plus logiquement de la stricte application du droit positif aux faits soient celles du juge-robot, tandis que celles du juge-humain seraient dictées par des considérations extérieures, souvent implicites. Faut-il, dès lors, suivre Jean Carbonnier lorsqu'il convenait que « le juge est un homme et non une machine à syllogismes : autant qu'avec sa connaissance des règles et sa

\footnotetext{
${ }^{1}$ N. BobBio, Essais de théorie du droit, trad. Ch. Agostini, M. Guéret, LGDJ-Bruylant, coll. La pensée juridique, 1998 , p. 38.

${ }^{2}$ E. PATTARO, « Définir le droit », Droits $1990, \mathrm{n}^{\circ} 11$, p. 47.

${ }^{3}$ On distingue le réalisme américain et le réalisme scandinave en raison des origines géographiques de ces courants de pensée, mais il existe bien des auteurs qui adhèrent à l'un ou à l'autre en n'étant ni américains ni scandinaves, y compris en France. Et des théoriciens du droit des États-Unis s'inscrivent davantage dans le courant dit «scandinave » que dans le courant dit « américain » du réalisme.
} 
logique, il juge avec son intuition et sa sensibilité $»^{1}$ ? L'algorithme, lui, est une machine à syllogismes. Quant au juge-humain, il semble que la connaissance des règles et la logique soient premières et que l'intuition et la sensibilité ne jouent qu'un rôle complémentaire, influençant l'orientation des décisions de justice seulement dans les cas les plus difficiles, face auxquels le juge électronique peine à s'accorder avec le juge réel.

Des robots utilisant des logiciels de reconnaissance vocale et sémantique pourraient-ils être les greffiers de demain ? C'est tout le service public de la justice que les nouvelles technologies de traitement de l'information invitent à repenser, à moderniser, mais non sans s'interroger quant à l'opportunité, aux bénéfices et aux risques de pareil renouvellement technologique dont les conséquences seraient telles qu'elles amèneraient peut-être la justice à changer d'ère, posant les jalons d'une nouvelle culture judicaire ${ }^{2}$. En ces pages, après avoir souligné, à l'aune des enseignements fournis par l'expérience de justice algorithmique, combien les décisions de justice ne paraissent pas devoir s'analyser tels les fruits de purs syllogismes et encore moins tels les fruits d'un entier libre arbitre des juges (II), sera posée plus concrètement la question d'une justice du futur abandonnée en tout ou partie à des machines « intelligentes » (III). Mais, en premier lieu, il convient de présenter plus finement l'expérience scientifique menée par les chercheurs britanniques et américains et ayant abouti à la création d'un algorithme capable de rendre les mêmes décisions de justice que les magistrats humains dans huit cas sur dix $(I)$.

\section{Présentation de l'expérience de justice algorithmique}

Un algorithme est une suite finie et précise d'opérations dont l'application permet de résoudre des problèmes, d'exécuter des tâches ou d'obtenir des résultats. Il fonctionne à partir d' «entrées » (les données initiales) et aboutit à des «sorties » (les résultats) en suivant différentes étapes qui requièrent des décisions logiques, des comparaisons ou des analogies. Les algorithmes sont à la base de l'informatique ; ils s'expriment le plus souvent à travers des programmes exécutables par ordinateur. Souvent assimilés à des «formules magiques », les plus connus sont ceux des moteurs de recherche, qui permettent, en fonction des mots-clés saisis par l'utilisateur, d'accéder aux pages web les plus pertinentes ${ }^{3}$. Des algorithmes servent aussi à traduire des textes, à classer des documents, à effectuer des calculs complexes, à crypter et décrypter des informations etc. En somme, les algorithmes semblent capables de

\footnotetext{
${ }^{1}$ J. CARBonnIER, Droit civil-vol. I : Introduction, Puf, coll. Quadrige, 2004, p. 23.

${ }^{2}$ C'est ainsi, par exemple, que l'avocat, ancien Garde des Sceaux et ancien Président du Conseil constitutionnel Robert Badinter en vient à déclarer : « Je suis admiratif quand j'assiste aux concours d'éloquence entre jeunes avocats parce qu'ils pratiquent un art en voie de disparition. Bientôt, l'éloquence n'aura plus sa place, sauf exception, dans la vie judiciaire. Elle ne servira plus à rien. Tout se passera par ordinateur. Un autre monde judiciaire est à venir. Et on n'y échappera pas parce que la massification des affaires et le manque de moyens obligent à aller dans cette direction là. La révolution du numérique me paraît aussi riche de perspectives pour la justice, mais aussi de dangers, en tout cas de profondes transformations structurelles que la révolution de l'imprimerie a pu l'être. La justice de demain n'aura plus rien à voir avec celle d'hier » (extrait de conférence entendu dans «L'intelligence artificielle peut-elle rendre justice?», C'est pour aujourd'hui ou pour demain, France inter, 12 août 2017).

${ }^{3}$ L'algorithme de Google est ainsi l'une des choses qui possèdent aujourd'hui le plus de valeur au monde.
} 
tout. Mais pourraient-ils aller jusqu'à rendre la justice à la place des hommes ou, du moins, avec les hommes ? Telle est la question posée par l'expérience commentée en ces lignes.

L'ordinateur, dénué de libre arbitre, exécute mécaniquement les opérations qui constituent l'algorithme. C'est pourquoi, si des chercheurs sont parvenus à élaborer un algorithme permettant à l'ordinateur de rendre des décisions de justice comme le feraient des magistrats humains, il est tentant d'en revenir à l'idée de «juge automate ${ }^{1}$. Tant l'algorithme que le syllogisme juridique seraient comparables à des recettes de cuisine : en mélangeant différents ingrédients précisément identifiés d'une manière bien comprise, tout cuisinier quel qu'il soit obtiendra le même plat ; tout juge quel qu'il soit aboutira à la même solution.

En l'occurrence, les scientifiques britanniques et américains se sont concentrés sur le cas de la Cour européenne des droits de l'homme, qui juge les États ayant potentiellement enfreint la Convention européenne de sauvegarde des droits de l'homme et des libertés fondamentales. Le choix de ce sujet d'expérience présentait un avantage : les décisions de la Cour, outre qu'elles sont en nombre suffisant pour fournir un échantillon représentatif ${ }^{2}$, comportent souvent de nombreuses pages dans lesquelles tant les faits que les arguments des parties et que les dispositions juridiques pertinentes sont finement expliqués ${ }^{3}$. Pour chaque cas étudié, l'algorithme avait donc à sa disposition suffisamment de données, suffisamment d' «entrées» pour effectuer sa tâche en toute connaissance de cause ${ }^{4}$. Mais ce choix présentait également une difficulté : les juges de la Cour européenne des droits de l'homme sont connus pour leur tendance à préférer traiter les cas en fonction des enjeux et des circonstances, éventuellement à l'aune du contexte politique propre à l'État mis en cause ${ }^{5}$. La Cour ne s'arc-bouterait pas sur les textes de droit en vigueur et privilégierait une approche ouverte permettant à l'opportunité de rivaliser avec la légalité. Sa méthode est ainsi qualifiée d' «essentiellement casuistique $»^{6}$, de «dynamique, téléologique et finaliste ${ }^{7}$. Ses juges pouvant, en outre, exprimer leurs opinions dissidentes, la CEDH paraît se rapprocher davantage des hautes cours des pays de common law que de celles des pays de droit de tradition romano-germanique, dans lesquels le syllogisme pur est réputé davantage

${ }^{1}$ Et l'ordinateur animé par des algorithmes peut être qualifié de « robot», être doué d'intelligence dite « artificielle» capable de réaliser des actes habituellement propres aux humains, éventuellement plus rapidement, plus précisément et/ou plus efficacement qu'eux.

${ }^{2}$ Les chercheurs se sont concentrés sur trois articles de la Convention européenne de sauvegarde des droits de l'homme et des libertés fondamentales : l'interdiction de la torture et de tout traitement dégradant (article 3), le droit à un procès équitable (article 6) et le droit au respect de la vie privée (article 8) — le contentieux lié à ce dernier article étant en pleine recrudescence depuis quelques années du fait des nombreuses révélations sur les opérations d'espionnage et de surveillance.

${ }^{3}$ Selon l'article 74 du Règlement de la Cour, ses arrêts doivent comporter, notamment, « l'exposé de la procédure ; les faits de la cause ; un résumé des conclusions des parties ; les motifs de droit ».

${ }^{4}$ Cela pose la question de la possibilité de reproduire l'expérience à l'échelle de juridictions rendant des décisions plus laconiques.

${ }^{5}$ F. MATSCHER, «Idéalisme et réalisme dans la jurisprudence de la Cour européenne des droits de l'homme », in Mélanges R. Ryssdal, Carl Heymanns Verlag, 2000, p. 881 s.

${ }^{6}$ F. MATSCHER, «Les contraintes de l'interprétation juridictionnelle », in F. SUDRE, dir., L'interprétation de la Convention européenne des droits de l'homme, Nemesis-Bruylant, 1998, p. 15.

${ }^{7}$ F. OsT, «Originalité des méthodes d'interprétation de la Cour européenne des droits de l'homme », in M. Delmas-Marty, dir., Raisonner la raison d'État, Puf, 1989, p. 405 ; F. SUDRE, « À propos du dynamisme interprétatif de la Cour européenne des droits de l'homme », JCP G 2001, I, p. 335. 
s'épanouir. De ce point de vue, les performances de l'algorithme sont remarquables. Et elles indiquent que l'activité juridictionnelle de la Cour européenne des droits de l'homme suit peut-être plus de lignes directrices claires et stables qu'on l'imagine parfois.

En effet, ainsi que le décrivent les chercheurs dans leur article publié le 24 octobre 2016, le juge-robot qu'ils ont créé est parvenu à deviner $79 \%$ des décisions de la Cour européenne des droits de l'homme. Pour parvenir à ce résultat, ils ont dû procéder à un vaste travail de documentation afin de déterminer les principales constances dans les arrêts de la Cour, établir les liens de corrélation montrant l'influence déterminante de certains éléments sur le verdict final et pouvoir ainsi fournir à l'algorithme des modèles censés le rendre le plus fiable possible. Par conséquent, si, dans $21 \%$ des situations, les juges ont tranché différemment de ce que le robot a supposé, peut-être est-ce parce qu'ils n'ont pas suivi les modèles.

En outre, l'algorithme est «auto-apprenant»: il se perfectionne à mesure qu'il est utilisé grâce à des modèles d'analyse sémantique appelés « N-grammes » ${ }^{1}$. Aussi semble-t-il logique qu'un tel outil produise des résultats significatifs en présence d'un langage très normé, calibré et systématique tel que le langage du droit et de la justice. L'algorithme repère les tendances textuelles qui mènent à des issues prévisibles en termes de violation ou de nonviolation de la Convention européenne de sauvegarde des droits de l'homme et des libertés fondamentales. Il existerait donc bien des liens de corrélation étroits entre les éléments fournis durant le procès et la décision finale des juges. En particulier, les chercheurs ont remarqué que des faits similaires aboutissent généralement à des décisions identiques, ce qui est heureux sous l'angle de la sécurité juridique, de la prévisibilité du droit, ainsi que de la confiance en la justice, et explique que le juge-robot soit parvenu à anticiper huit fois sur dix les verdicts rendus par la Cour européenne des droits de l'homme.

Au total, 584 décisions ont été analysées, contenant à parts égales des cas ayant abouti à une condamnation et des cas ayant abouti à une non-condamnation, cela afin d'éviter tout biais dans l'instruction de l'apprenti juge-robot. Concrètement, les scientifiques ont fourni à l'algorithme les motifs de chacun des arrêts de la Cour européenne des droits de l'homme (faits, procédure antérieure, arguments présentés par les parties et règles de droit pertinentes, c'est-à-dire éventuellement applicables). Ensuite, l'algorithme devait deviner le dispositif, donc découvrir si la Cour a condamné ou non l'État mis en cause. Pareille méthode $a$ posteriori pose la question de la possibilité de créer des juges-robots intervenant a priori, car qui alors leur présenterait les faits et, surtout, rechercherait les normes juridiques potentiellement mobilisables ? Il faudrait que le juge-robot soit capable d'effectuer ces tâches, mais les capacités de celui élaboré par les chercheurs britanniques et américains sont beaucoup plus limitées. Pour que les juges-robots puissent être autonomes, il faudrait que l'informatique progresse encore d'un ou même de plusieurs niveaux. Pour l'heure, seuls des juges-robots sous tutelle humaine sont concevables.

De plus, l'algorithme des scientifiques anglo-saxons se contente de répondre à une alternative binaire simple : condamner ou ne pas condamner, reconnaître une violation ou ne pas reconnaître de violation d'un article de la Convention européenne de sauvegarde des

\footnotetext{
${ }^{1}$ Les $\mathrm{N}$-grammes servent à déterminer, en présence d'un certain nombre de mots ou de lettres, quel est le mot ou la lettre qui a la plus grande probabilité d'y être associé.
} 
droits de l'homme. Il n'en demeure pas moins que cet algorithme a rendu dans huit cas sur dix la même décision que les magistrats humains et que cela interroge, spécialement sous l'angle de la pensée juridique et plus encore sous l'angle du jusréalisme, courant théorique qui voit le droit principalement, si ce n'est uniquement, dans les activités des tribunaux et qui est de plus en plus populaire - à tel point qu'il concurrence désormais le normativisme kelsénien dans lequel les juges sont de purs organes exécutifs se bornant à appliquer très systématiquement la loi qui leur est donnée aux cas qui leur sont soumis, les jugements contenant seulement des normes individuelles situées tout en bas de la «pyramide» des normes.

\section{Les enseignements théoriques de l'expérience : le « réalisme juridique » en question}

Qu'un juge-robot soit en mesure de trouver huit fois sur dix quelles décisions les magistrats de la Cour européenne des droits de l'homme ont prises montre que la justice est évidemment loin d'être rendue au hasard, mais aussi qu'elle n'est guère à la merci de considérations extra-juridiques cachées ou sous-entendues $(A)$. En même temps, que deux fois sur dix les décisions du juge-robot et du juge-humain divergent est également significatif ${ }^{1}$. C'est pourquoi l'analyse la plus conforme à la réalité judiciaire paraît être celle proposée par le réalisme scandinave et plus particulièrement par la théorie des contraintes juridiques : il est possible et en même temps difficile de prévoir les décisions des tribunaux car, si la première des contraintes qui orientent le juge-robot autant que le juge-humain réside dans le droit positif, d'autres, plus subjectives, plus insidieuses et auxquelles l'algorithme ne saurait en revanche être soumis, peuvent également entrer en jeu $(B)$.

\section{A. $79 \%$ d'accords entre le juge-humain et le juge-robot : la part de logique de la justice?}

Si la décision de justice était simplement le résultat d'un parfait syllogisme, si elle jaillissait naturellement du mélange des faits et du droit, il ne serait guère difficile d'élaborer un algorithme capable de rendre la justice. Seuls les cas les plus complexes, notamment lorsque la qualification des faits est rendue pénible par leur situation à mi-chemin entre deux régimes juridiques, poseraient problème. Or longtemps, en France du moins, les théories de l'interprétation ont sacralisé la loi. La doctrine légaliste de la seconde moitié du XIX ${ }^{\mathrm{e}}$ s., dite « de l'Exégèse », considérait ainsi que chaque disposition légale ne possèderait qu'un seul et unique sens et que le rôle du juge serait seulement de l'identifier. L'interprétation de la loi

\footnotetext{
${ }^{1}$ Cela d'autant plus que, dans les situations où l'algorithme n'a pas saisi les motivations amenant les juges à trancher dans un sens plutôt que dans un autre, l'alternative s'offrant à lui étant binaire, sa position aura néanmoins été la même que celle de la Cour européenne des droits de l'homme dans $50 \%$ des cas. Ainsi, si le juge-humain se prononce sous l'influence d'un facteur $x$ et le juge algorithmique sous l'influence d'un facteur $y$, l'un et l'autre tomberont néanmoins d'accord au moins une fois sur deux. Cela signifie qu'il faut multiplier par deux les $21 \%$ d'erreurs pour obtenir le taux de prédictions véritablement bien-fondées du juge-robot. Celui-ci n'est alors que de $58 \%$.
} 
comme celle des faits seraient purement déclaratives et en aucun cas constitutives, seraient les fruits d'actes de connaissance et non d'actes de volonté. Cette conception est logique dès lors que les fonctions de création et d'application du droit sont clairement identifiées et séparées dans l'État : le législateur crée, le juge applique.

Cependant, les faits ont largement contredit la vision d'un juge « esclave» du législateur, «bouche» de la loi ou «bras »du Parlement, simple exécutant sans marge de manœuvre se bornant à mettre en œuvre la méthode du syllogisme tel un automate. Au début du $\mathrm{XX}^{\mathrm{e}}$ s., l'École de la libre interprétation, emmenée par François Gény et considérant que les juges pourraient très bien «juger la loi » et faire prévaloir d'autres considérations par rapport au droit positif, a pris le pas sur celle de l'Exégèse. Avec cette libre recherche scientifique, le bon juge ne serait plus celui qui applique scrupuleusement la loi, émanation de la volonté du peuple souverain, mais celui qui l'adapte à l'état actuel de la société et aux spécificités de chaque litige, cela en recourant à des «méthodes scientifiques » permettant de prendre en compte les données sociales, morales, historiques ou encore économiques et de pondérer les intérêts en présence ${ }^{1}$. En somme, les magistrats pourraient — et ils ne s'en priveraient pas — réécrire arbitrairement la loi, jusqu'à s'en détacher de fait, afin d'adapter leurs décisions aux besoins de l'espèce et/ou aux besoins du temps présent.

L'expérience de justice algorithmique ne contredit pas la vision d'un juge libre, mais elle montre que, s'il est libre, le juge n'en profite pas pour rendre le droit à sa guise et préfère généralement s'appuyer sur les plus solides arguments issus du droit positif ${ }^{2}$. En effet, l'algorithme, pour sa part, ne disposait que d'éléments objectifs, à savoir les faits, la procédure antécédente, les arguments des parties et les dispositions de droit positif pertinentes. Or, à l'aune de ces seules informations, il est parvenu à deviner dans la majorité des situations quelle avait été la décision retenue par les magistrats de la Cour européenne des droits de l'homme.

Plus encore, les résultats de l'expérience permettent de relativiser les propositions des théoriciens du droit rattachés au réalisme américain. Et, puisque ces auteurs défendent des thèses plus ou moins radicales, loin de suivre une doctrine précisément établie, ces résultats, pour le dire caricaturalement, vont surtout à l'encontre des plus radicales, tandis qu'ils confortent en partie les plus modérées, qui se rapprochent du réalisme scandinave.

Pour ce qui est des thèses semble-t-il les plus excessives par rapport à la réalité des pratiques judiciaires, d'aucuns, à la suite du philosophe pragmatiste John Dewey, considèrent que, dans l'ordre empirique des choses, les juges, dans un premier temps, trancheraient en opportunité les cas, en fonction de leurs conséquences pour les parties mais aussi de leurs conséquences pour l'ordre social, de leurs conséquences économiques, de leurs conséquences

\footnotetext{
${ }^{1}$ François Gény écrivait en ce sens que la méthode du juge devrait consister «à reconnaître les intérêts en présence, à évaluer leurs forces respectives, à les peser, en quelque sorte, avec la balance de la justice, en vue d'assurer la prépondérance des plus importants, d'après un criterium social, et finalement d'établir entre eux l'équilibre éminemment désirable » (F. GÉNY, Méthodes d'interprétation et sources en droit privé positif, t. I, $2^{\mathrm{e} e ́ d ., ~ L G D J, ~ 1919, ~ p . ~ 167) . ~}$

${ }^{2}$ Bien sûr, le propos développé en ces lignes concerne essentiellement les juges appartenant aux cours de dernier ressort - ce qu'est la Cour européenne des droits de l'homme —, les juges appartenant aux tribunaux ou aux cours d'appel étant pour leur part forcément influencés par le risque de voir leurs jugements ou arrêts contredits en cas d'appel ou de pourvoi.
} 
symboliques etc., puis, dans un second temps, chercheraient au sein du droit positif disponible quelques moyens de justifier juridiquement mais plus ou moins artificiellement les décisions prises ${ }^{1}$. Ainsi Oliver Wendell Holmes se félicitait-il du «mérite du common law [qui] est de décider du cas d'abord et de déterminer les principes après coup $»^{2}$. Il décrivait : «First we decide, then we deduce » ${ }^{3}$, donc «tout d'abord nous décidons, puis nous justifions notre décision ». S'il y a syllogisme, celui-ci ne serait pas déductif comme le suppose la logique formelle normativiste, mais «inductif et pragmatique ${ }^{4}$ en ce qu'il consisterait à choisir d'abord le sens de la décision de justice pour ensuite l'habiller de quelques "supports d'imputation $»^{5}$ - en piochant dans le bagage de lois et règlements, en particulier dans leurs nombreuses notions à contenu variable, et en interprétant ces dispositions en faveur de la solution retenue ${ }^{6}$. Ce ne serait donc pas la décision qui dériverait de la règle de droit mais la règle de droit qui dériverait de la décision. Telle est l'une des principales idées du réalisme américain, la validité et la hiérarchie des normes étant appréhendées telles des propositions métaphysiques à écarter purement et simplement du raisonnement. Dès lors, le droit positif serait malléable, incertain et imprévisible.

L'expérience des chercheurs anglo-saxons ne permet pas de se prononcer quant à la pertinence de pareille conception du travail des magistrats puisque l'algorithme s'est basé sur les données issues des arrêts et donc sur les arguments et explications retenus par les juges eux-mêmes. Néanmoins, le grand nombre d'arrêts rendus à l'unanimité, i.e. sans opinion dissidente, et la précision des motifs fournis par la Cour européenne des droits de l'homme, les règles de droit mobilisées étant loin de se borner à jouer un «rôle décoratif pour répondre à l'exigence de motivation des décisions de justice et garantir un minimum d'acceptabilité de la décision de justice par ses destinataires ${ }^{7}$, supposent que cette juridiction s'appuie sur le syllogisme normativiste, logique et déductif, et non sur le syllogisme réaliste, régressif et inversé.

${ }^{1}$ J. Dewey, «Logical Method and Law », Philosophical Review 1924, p. 560 s.

${ }^{2}$ O. W. HOLMES, op. cit., p. 81.

${ }^{3}$ Cité par R. DANISCH, Pragmatism, Democracy, and the Necessity of Rhetoric, University of South Carolina Press, 2007, p. 13.

${ }^{4}$ F. Ost, Dire le droit, faire justice, Bruylant, coll. Penser le droit, 2007, p. 104.

${ }^{5}$ G. TIMSIT, «Raisonnement juridique », in D. Alland, S. RIALS, dir., Dictionnaire de la culture juridique, Lamy-Puf, coll. Quadrige-dicos poche, 2003, p. 1295.

${ }^{6}$ Cette conception a rencontré un certain succès et, en France, y compris le Commissaire du gouvernement Chenot a pu soutenir que « l'essentiel est le dispositif, les motifs sont construits après » (B. CHENOT, «L'existentialisme et le droit », Revue française des sciences politiques 1953, p. 57 (cité par G. THUILLIER, « Probabilisme et art de juger », in D. Alland, S. Rials, op. cit., p. 1216)). Quant à Jean Carbonnier, il enseignait que « tous nos juges jugent d'abord en équité, puis habillent leur équité d'un revêtement de droit logique. On n'a pas une compréhension exacte du droit si l'on ne se dit pas que, bien souvent, le juge, et même le juge au niveau de la Cour de cassation, commence par se demander à qui, en équité, il doit donner raison, quitte ensuite à trouver les moyens logiques de lui donner raison. Le processus de l'analyse dogmatique qui montre le juge descendant de la règle de droit vers la décision est bien souvent mis en défaut. En réalité, le juge commence toujours par peser la décision, puis remonte ensuite à la règle de droit. Et comme il y a beaucoup de règles de droit possibles [à partir] d'une même règle, il arrivera toujours, une fois qu'il aura posé sa décision, à découvrir une règle de droit satisfaisante » (J. CARBONNIER, Théorie sociologique des sources du droit, Association corporative des étudiants en droit de l'Université Panthéon-Sorbonne, 1961, p. 181-182).

${ }^{7}$ X. Magnon, Théorie(s) du droit, Ellipses, coll. Universités-Droit, 2008, p. 133. 
Toujours est-il que l'expérience de justice robotisée conduit à contredire dans une certaine mesure - non complètement tant les $21 \%$ de cas dans lesquels les approches du juge-robot et du juge-humain diffèrent posent question - le jusréalisme américain sous l'angle des sources du droit. En effet, ses tenants considèrent que les décisions juridictionnelles seraient davantage dictées par des éléments subjectifs, très contingents et aléatoires, que par les faits, le droit positif et leur réunion à l'aide du syllogisme. Selon eux, il faudrait « rechercher l'origine des décisions dans les conversations du juge avec les autres membres de la juridiction, dans sa lecture des journaux et dans le souvenir de l'enseignement qui lui a été prodigué à l'université ${ }^{1}$. Sceptiques à l'égard de la logique juridique déductive comme clé de compréhension de l'activité des tribunaux, les jusréalistes américains s'emploient à saisir les ressorts « réels » de cette activité. Ils mettent en avant les motivations ni juridiques ni casuelles des décisions de justice telles que les croyances des juges, leurs préjugés, leurs habitudes ou leurs préférences en termes de justice sociale ${ }^{2}$, autant de données qu'un algorithme ne saurait deviner et par suite intégrer.

Oliver Wendell Holmes écrivait, en 1881, afin de motiver son rejet du syllogisme, que «la vie du droit n'est pas logique ; elle est expérience ${ }^{3}$. Le juge-robot étant sensible à la logique mais non à l'expérience, il faut croire que la vie du droit, en tout cas pour ce qui est de son pan juridictionnel, est davantage faite de logique que d'expérience. Quant à Karl Llewellyn, l'un des plus illustres réalistes américains, il estimait que le droit serait surtout « une idéologie et un ensemble d'idées envahissantes et puissantes qui sont largement non verbalisées, largement implicites, et qui passent inaperçues ${ }^{4}$. À nouveau, si les jugements et arrêts découlaient véritablement de telles considérations non verbalisées, implicites et inaperçues, il aurait été vain d'entreprendre l'élaboration d'un algorithme capable de deviner le sens de leurs dispositifs.

Certains sont allés jusqu'à soutenir que les juges n'obéiraient qu'à leur instinct, qu'à leur intuition, qu'à leur opinion, ou encore que jusqu'aux contenus de leurs repas, leur plus ou moins grand épanouissement dans leurs vies personnelles ou même l'heure de la journée ou le fait d'être en été ou en hiver influenceraient leurs activités juridictionnelles ${ }^{5}$. Ces thèses

${ }^{1}$ F. S. CoHEn, The Legal Conscience - Selected Papers, Yale University Press, 1960 (cité par F. Michaut, La recherche d'un nouveau paradigme de la décision judiciaire à travers un siècle de doctrine américaine, L'Harmattan, coll. Logiques juridiques, 2001, p. 211).

${ }^{2}$ Par exemple, le juge Benjamin Cardozo a promu une méthode de l'utilité sociale devant amener à juger en fonction des conséquences (sociales, économiques, politiques, morales) des décisions et non en fonction du droit positif.

${ }^{3}$ O. W. Holmes, The Common Law, MacMillan, 1881, p. 1.

${ }^{4}$ K. Llewellyn, My Philosophy of Law, Boston Law Co., 1941, p. 183.

${ }^{5}$ Le plus radical parmi les jusréalistes américains fut peut-être Jerome Frank, de l’École de droit de l'Université de Chicago. Ce professeur dénonçait l'illusion du « jeu intellectuel » consistant à prétendre identifier des règles dans la loi alors que la pratique réelle des cours et tribunaux dépendrait de tout autres données; à tel point qu'une décision de justice serait davantage influencée par le " petit déjeuner du juge » que par le contenu de la loi (J. Frank, Courts on Trial - Myth and Reality in American Justice, Princeton University Press, 1950). Appliquant les thèses freudiennes au droit, il proposa l'équation « $D=P \times S », D$ étant la décision de justice, $P$ la personnalité du juge et $S$ les stimuli qui le frappent. Le droit, en pratique, serait donc le résultat de la personnalité du magistrat qui le prononce - elle-même conséquence de «ses parents, ses études, ses professeurs, ses condisciples, sa femme, ses enfants [et] les livres lus » (ibid.) - et des évènements contingents qui influencent son humeur le jour où il le prononce (par exemple, la qualité de son repas, le fait d'être le matin ou l'après-midi, le fait d'être avant ou après les vacances (ibid.)). Si telles étaient de facto les sources du droit, il 
tendent a fortiori à être démenties par l'expérience de justice algorithmique ; et il faut supposer que les erreurs dans les prédictions du juge-robot sont moins imputables à l'entrée en jeu de telles considérations extrêmement subjectives et parfois même insensibles qu'à des éléments tels que l'obédience politique ou la volonté de faire prévaloir certains intérêts plutôt que d'autres, même si tous sont inavouables et donc inaccessibles au juge algorithmique. Jean Carbonnier pouvait relever combien « il y a toujours eu des jugements intuitifs $»^{1}$; mais, pour les jusréalistes les plus radicaux, il n'y aurait que cela ${ }^{2}$. Les $79 \%$ de concordances entre les arrêts réels de la CEDH et les arrêts supposés par l'algorithme incitent à penser qu'il peut exister des jugements intuitifs, mais aussi que, le plus souvent, les jugements sont la conséquence de véritables syllogismes et que, de fait, les juges ne jouissent pas d'une totale liberté d'interprétation ni d'action. Et les $21 \%$ de divergences entre les arrêts réels de la $\mathrm{CEDH}$ et les arrêts supposés par l'algorithme invitent à imaginer que d'autres éléments que les seuls faits et dispositions juridiques pertinentes influencent parfois de manière déterminante les choix opérés par les magistrats ${ }^{3}$.

\section{B. $21 \%$ de désaccords entre le juge-humain et le juge-robot : la part d'arbitraire de la justice?}

Les auteurs de l'expérience de justice algorithmique expliquent que leur algorithme serait parvenu plus aisément à deviner les issues des procès à l'aune de leurs données factuelles qu'à l'aune de leurs données juridiques. Ils en déduisent que cela conforterait les conceptions des réalistes américains en prouvant que les décisions de justice seraient davantage dépendantes de considérations factuelles que de considérations juridiques ${ }^{4}$. Cependant, cela revient à réduire excessivement le propos de la théorie réaliste tant celle-ci ne considère guère que les faits déterminants seraient uniquement les faits des espèces, bien au contraire. Surtout, il semble, sous l'angle de la pensée juridique, que d'autres leçons doivent être tirées des résultats de l'expérience.

En effet, il est logiquement impossible de savoir si la Cour européenne des droits de l'homme condamnera ou ne condamnera pas un État à l'aune du seul droit potentiellement applicable ; car tout dépend des faits en cause et de l'applicabilité ou non-applicabilité de ce

aurait été difficile de créer un algorithme capable de prédire la majorité des décisions de justice. D’ailleurs, Jerome Frank imaginait impossible toute anticipation des décisions de justice (ibid.). Mais la bonne équation n'est peut-être pas «D $D=P \times S »$ mais plutôt « $D=L \times P \times S », L$ désignant la loi et jouant le rôle essentiel.

${ }^{1}$ J. CARBOnNIER, Théorie sociologique des sources $d u$ droit, op. cit., p. 41.

${ }^{2}$ Par exemple, J. C. Hutcheson, « The Judgment Intuitive: the Function of the "Huntch" in Judicial Decision », Cornell Law Quarterly 1929, p. 274 s.

${ }^{3}$ D'autant plus si l'on considère que ce sont $42 \%$ des prédictions qui ne sont pas fiables, ajoutant $21 \%$ de prédictions concordantes par hasard aux $21 \%$ de prédictions erronées (l'algorithme n'ayant à chaque fois le choix qu'entre deux options).

${ }^{4}$ Ils écrivent ainsi : « Notre analyse empirique indique que les faits à l'origine du litige sont le facteur prédictif le plus important. Ceci est cohérent avec la théorie du réalisme juridique suggérant que la prise de décision judiciaire est affectée de manière significative par le stimulus des faits. [...] Nos résultats semblent confirmer que les arrêts rendus par la CEDH se basent principalement sur des arguments non juridiques. [...] [Ils] vont dans le sens de la théorie réaliste selon laquelle les juges sont sensibles au non-droit plutôt qu'au droit » (N. Aletras, V. LAmpos, D. Tsarapatsanis, D. PreoțiUC-Pietro, art. préc.). 
droit à ces faits. Si l'algorithme sait seulement que le droit condamne les voleurs, comment pourrait-il deviner si M. X a été ou non condamné ? Il faut qu'il sache si M. X a volé ou non. En revanche, si l'algorithme a connaissance des faits et non du droit, il pourra deviner avec plus de chances de succès la décision des juges. Si M. X a volé et que, par le passé, M. Y, qui a volé, a été condamné et $\mathrm{M}$. Z, qui a volé, a été condamné, il en conclura logiquement que M. X a lui aussi été condamné sans pour autant que l'information selon laquelle le droit punit le vol lui ait été fournie. Mais de la loi éventuellement applicable on ne peut rien déduire, tout dépendant de son application effective ou non aux faits, donc des faits. Savoir que la loi L est la loi pertinente dans le cas A et qu'elle a déjà été la loi pertinente dans les cas B, C et D n'est guère utile afin de savoir si cette loi L a effectivement été appliquée dans le cas A. Que l'algorithme parvienne plus souvent à déterminer l'issue des procès à l'aune des faits des espèces qu'à l'aune du droit pertinent serait donc sans lien avec le fait que les juges soient davantage influencés par des considérations factuelles que par des considérations juridiques.

Il n'en demeure pas moins que l'expérience menée n'aboutit pas à faire totalement triompher le normativisme (et le formalisme qui l'accompagne) du réalisme (et du subjectivisme qui l'accompagne). Peut-être les $21 \%$ d'erreurs sont-ils plus intéressants que les $79 \%$ de réussites. Si la prise de décision juridictionnelle obéissait à une stricte logique formelle et déductive, les chercheurs seraient peut-être parvenus à créer un juge algorithmique s'accordant plus systématiquement avec le juge-humain. Aussi, entre les excès du réalisme américain et les insuffisances du normativisme kelsénien, peut-être est-ce la voie du réalisme scandinave qui permet le mieux de rendre compte de la réalité des mécanismes judiciaires.

Avec la version américaine du jusréalisme, la liberté des juges à l'égard des codes est un devoir ou, du moins, une réalité indiscutable allant de pair avec une ignorance de ces codes - a priori puisqu'ils peuvent ensuite être mobilisés afin de justifier des décisions déjà prises. Avec la version scandinave du jusréalisme, cette liberté est reconnue en même temps qu'est reconnue l'existence de contraintes juridiques étant, de facto, les données qui influencent le plus intimement les décisions juridictionnelles ${ }^{1}$. La théorie réaliste scandinave «admet que l'interprétation est juridiquement libre [...] [et] comprend qu'elle est soumise à un déterminisme $»^{2}$. Alf Ross, l'un des principaux promoteurs de ce réalisme «doux» ou « modéré », notait que «le droit présuppose non seulement que le juge ait un comportement régulier, mais aussi qu'il ait le sentiment d'être lié par les règles ${ }^{3}$ — on peut être libre, n'encourir aucun jugement ni aucune sanction, et néanmoins être animé par quelques convictions telles que celle selon laquelle appliquer la loi serait la première des missions d'un juge. Et Alf Ross ne niait en aucun instant la prégnance de l' «idéologie de la hiérarchie des normes ${ }^{4}$, tout en y voyant donc une idéologie.

${ }^{1}$ V. Champeil-Desplats, C. Grzegorczyk, M. Troper, dir., Théorie des contraintes juridiques, BruylantLGDJ, coll. La pensée juridique, 2005.

${ }^{2}$ M. TROPER, «La liberté d'interprétation du juge constitutionnel », in P. AMSELEK, dir., Interprétation et droit, Bruylant, 1995, p. 242.

${ }^{3}$ A. Ross, On Law and Justice, Stevens \& Sons, 1958, p. 34 (cité par Ch. GrZEGORCZYK, F. Michaut, M. TROPER, Le positivisme juridique, LGDJ, coll. La pensée juridique, 1993, p. 325).

${ }^{4}$ Ibid., p. 54 (cité par F. OST, M. VAN DE KeRCHOVE, De la pyramide au réseau? - Pour une théorie dialectique du droit, Publications des Facultés universitaires Saint-Louis, 2002, p. 381). 
Si le juge est souverain ou quasi-souverain, son action n'en est pas moins sous l'influence de divers éléments ; et ces éléments, loin d'être subjectifs et cachés, parfois même inconscients, comme le postulent les réalistes américains, sont davantage objectifs et assumés, a fortiori dès lors qu'il s'agit simplement des lois, règlements et jurisprudences applicables. Au cours de l'expérience, l'algorithme pouvait par conséquent reconnaître ces contraintes juridiques et, en les confrontant aux faits de chaque espèce, deviner dans quel sens la Cour avait tranché. Mais les contraintes qui orientent les comportements des juges malgré leur liberté théorique ne se limitent pas aux contraintes juridiques, bien que celles-ci soient les plus importantes, et d'autres, plus insidieuses et relevant de l'arbitraire de chaque magistrat, peuvent dans certains cas jouer un rôle déterminant, expliquant la part d'erreurs dans les prédictions du juge algorithmique. De plus, en raison de la «texture ouverte du droit ${ }^{1}$, nul doute que le juge-robot et le juge-humain sont parvenus plus aisément à s'accorder concernant «les cas centraux et clairs auxquels la règle s'applique avec certitude $»^{2}$ que concernant «les cas pour lesquels il existe des raisons aussi bien d'affirmer que de nier qu'elle s'y applique $»^{3}$.

Les origines des divergences entre les décisions du juge-robot et du juge-humain sont sans doute multiples ; et il est difficile de ne pas y inclure la subjectivité et la personnalité de chaque magistrat. Si les juges-humains sont tantôt sujets des règles de droit (ce qui correspond à la position normativiste) et tantôt acteurs des règles de droit (ce qui correspond à la position réaliste), un juge-robot ne saurait qu'être sujet des règles de droit — on peut imaginer un ordinateur appliquer grâce à la logique du syllogisme des normes à des faits, mais il est plus difficile de l'imaginer décider de quelques orientations jurisprudentielles ou opérer quelques interprétations audacieuses des termes de la loi.

C'est bien sous les traits d'un «casse-tête » que le comportement judiciaire se présente ${ }^{4}$, loin de se réduire au modèle normativiste ou au modèle réaliste ${ }^{5}$. Dans leur article, les chercheurs indiquent que «l'algorithme a eu tendance à fournir des jugements erronés quand il s'est trouvé en présence de deux cas similaires ayant entraîné une condamnation et une noncondamnation $»^{6}$. Lorsque des faits identiques ou du moins proches sont traités différemment en fonction des juges à qui ils sont soumis, l'algorithme ne peut que s'y perdre et le réalisme, y compris dans sa version la plus radicale, ne peut qu'en sortir conforté. Néanmoins, dans la majorité des cas, les situations proches ont été traitées identiquement. Ce sont alors le syllogisme et le normativisme qui se trouvent renforcés. Qui observe la pratique des juges, audelà de l'expérience de justice algorithmique, constate combien ils expriment généralement le sentiment d'être liés par les lois et combien la jurisprudence revêt une cohérence suffisante pour que les décisions soient loin d'être imprévisibles. Dire qu'il existe une liberté du juge, ce n'est pas — et c'est heureux — dire qu'il existerait une obligation pour le juge de statuer contra legem ou para legem. La théorie des contraintes juridiques explique pourquoi cette liberté n'entraîne pas une parfaite cacophonie juridictionnelle.

\footnotetext{
${ }^{1}$ H. L. A. HART, Le concept de droit, trad. M. van de Kerchove, Publications des Facultés universitaires SaintLouis, 1994, p. 155.

${ }^{2}$ Ibid.

${ }^{3}$ Ibid.

${ }^{4}$ L. BAUM, The Puzzle of Judicial Behavior, University of Michigan Press, 2009.

${ }^{5}$ B. LEITER, «Legal Formalism and Legal Realism: What is the Issue? », Legal Theory 2010, n 16 , p. $111 \mathrm{~s}$.

${ }^{6}$ N. Aletras, V. LAmpos, D. Tsarapatsanis, D. Preoțiuc-Pietro, art. préc.
} 
Pour autant, si les 584 dossiers objets de l'expérience devaient être aujourd'hui examinés à nouveaux frais par d'autres juges, le taux de correspondance avec les premières décisions serait peut-être plus proche de $79 \%$ que de $100 \%$. La marge d'erreur du juge-robot, soit $21 \%$, ne signifie pas nécessairement qu'il se trompe mais plutôt qu'il dit différemment le droit que le juge-humain. Ensuite, faut-il davantage faire confiance au premier ou bien au second ? Il est sans doute difficile de préférer une justice des machines à une justice des hommes, quels que soient les mérites respectifs de l'une et de l'autre. Néanmoins, les derniers progrès des technologies de traitement de l'information, dont l'expérience commentée en ces lignes témoigne, posent la question, si ce n'est d'une justice rendue par ordinateur, du moins d'une justice assistée par ordinateur. La « justice prédictive »- puisque telle est l'expression que la doctrine semble vouloir consacrer - interroge les évolutions technologiques du service public de la justice, évolutions qui, si elles deviennent possibles à mesure que des algorithmes performants sont élaborés, ne sont pas forcément indispensables ni souhaitables.

\section{Les prolongements prospectifs de l'expérience : la « justice prédictive » en question}

L'économie et les pratiques du droit et de la justice pourraient être impactées de façon croissante par les nouveaux services permis par le perfectionnement de l'intelligence artificielle. Ne sont-ce que les prémices de profonds bouleversements technologiques des professions juridiques qui se laissent aujourd'hui ressentir — et dont attesterait l'expérience réalisée par les scientifiques anglo-saxons ? Cette expérience ne se contente pas de fournir quelques enseignements à la théorie juridique ; elle appelle également des prolongements dans la pratique juridique, c'est-à-dire la mise en œuvre effective de solutions de justice algorithmique. La manière de rendre la justice mais aussi la manière de défendre et de conseiller les justiciables pourraient être affectées profondément par l'irruption de ces nouvelles technologies au sein des prétoires et des cabinets; et cela dans un futur qu'il n'est pas nécessaire d'imaginer lointain. Déjà les cours d'appel de Rennes et de Douai, comme le barreau de Lille, testent un logiciel de justice prédictive ${ }^{1}$. Ainsi l'expérience précédemment présentée et, plus généralement, les récents développements autour de la justice prédictive amènent-ils à interroger la possibilité et l'opportunité de l'éventuelle automatisation ou semiautomatisation des fonctions de magistrat $(A)$ et d'avocat $(B)^{2}$.

\footnotetext{
${ }^{1}$ Il s'agit de celui proposé par la société Predictice, expérimenté durant le printemps 2017 afin de mesurer les apports qui pourraient être les siens.

${ }^{2}$ En France, les commentaires et les réflexions autour de la justice algorithmique se sont multipliés au cours de l'année 2017 : v. not. A. GARAPON, «Les enjeux de la justice prédictive », JCP G 2017, p. 47 s. ; B. DONDÉRO, «Justice prédictive : la fin de l'aléa judiciaire ?», D. 2017, p. 532 s. ; B. LAMON, « La profession d'avocat et la justice prédictive : un bel outil pour le développement du droit», D. 2017, p. 808 s. ; M. MEKKI, «If Code is Law, then Code is Justice? Droits et algorithmes », Gaz. Pal. 27 juin 2017, n 24, p. 10 s. ; S. LARRIÈRE, «Confier le droit à l'intelligence artificielle : le droit dans le mur?», RLDI 2017, $\mathrm{n}^{\circ} 134$, p. 38 s. ; M.C. LASSERRE, «L'intelligence artificielle au service du droit : la justice prédictive, la justice du futur ?», LPA 30 juin $2017, \mathrm{n}^{\circ} 130$, p. 6 s. ; ainsi que les actes du colloque «Justice prédictive : évolution, révolution?», organisé le 23 mai 2017 par la cour d'appel de paris (<ca-paris.justice.fr>).
} 


\section{A. Possibilité et opportunité de l'algorithmisation du travail des magistrats}

$\mathrm{Au}$ début du $\mathrm{XX}^{\mathrm{e}}$ s., le théoricien du droit américain Roscoe Pound dénonçait le caractère automatique de l'activité judiciaire dès lors qu'elle s'appuie indéfectiblement sur le syllogisme formaliste ${ }^{1}$. Aujourd'hui, cependant, rares sont ceux qui contestent que les magistrats, au moment de juger, peuvent ou même doivent ouvrir la porte à l'éthique et autres formes de droit non officiel, cela afin d'adapter leurs décisions aux particularités de chaque cas. De ce point de vue, la qualité de la justice diminuerait peut-être inexorablement si les juges-humains laissaient leur place à des juges-robots. En revanche, l'automatisation de la justice permettrait d'accélérer son cours et de diminuer ses coûts. Alors que certains tribunaux sont saturés par un trop grand nombre de dossiers à traiter, recourir aux juges-robots pourrait être, sous cet angle, salutaire.

En outre, le juge algorithmique - à condition qu'on parvienne technologiquement à créer des algorithmes suffisamment fiables - permettrait, pour chaque cas, d'obtenir la décision la plus exactement conforme au droit positif. Il serait ainsi un gage de sécurité juridique et permettrait d'uniformiser les décisions de justice, ce dont l'opportunité serait à tout le moins discutable. En jouant sur les réglages de l'algorithme, on pourrait également appliquer une politique judiciaire uniforme sur l'ensemble du territoire. Ensuite, la justice automatisée pourrait concerner non toutes les affaires mais seulement les plus simples ou celles se rapportant aux prétentions ou aux enjeux les moins importants. L'objectif serait de libérer les magistrats de la justice du quotidien. Il s'agirait donc essentiellement de désengorger les tribunaux et de permettre à la justice de gagner en célérité, en efficacité.

Nonobstant ces bénéfices potentiels, il est difficile d'imaginer, concernant la justice correctionnelle et la justice criminelle, comment des verdicts et des peines fixés par ordinateur pourraient être acceptés. Le jury d'assises n'est-il pas là non pour rendre une justice plus juste par rapport à celle des magistrats professionnels mais pour instiller dans l'esprit des accusés qu'ils sont jugés «par le peuple », qu'ils sont jugés « démocratiquement»? Aussi toutes les «legal start-up » qui investissent aujourd'hui le marché de la justice prédictive s'interdisentelles d'appliquer leurs outils à la matière pénale. Mais n'est-ce pas y compris au niveau des justices civile et administrative qu'il serait difficile d'imposer aux justiciables des jugements produits par des juges-robots? Un juge sans éthique, sans déontologie, sans responsabilité, sans humanité mériterait-il encore d'être appelé « juge »? La justice automatisée, peinant à prendre en considération les spécificités de chaque cas et à individualiser les décisions, pourrait difficilement ne pas déboucher sur des excès, sur une forme de totalitarisme potentiellement contraire à certains principes de l'État de droit.

Dans tous les cas, il semble que les technologies de traitement de l'information ne sont pas encore arrivées à un tel niveau de perfectionnement que la question du remplacement des hommes par des automates puisse raisonnablement être posée. Il serait peut-être temps, en revanche, de songer plus sérieusement aux modalités et aux limites d'une justice assistée par ordinateur, les outils informatiques ne servant plus seulement à enregistrer et stocker les informations, ainsi qu'à rédiger les documents, mais devenant de véritables aides à la prise de

\footnotetext{
${ }^{1}$ R. Pound, « Mechanical jurisprudence », Columbia Law Review 1908, p. 605 s.
} 
décision. L'enjeu est et sera sans doute moins de choisir entre juges-robots et juges-humains que de mettre les premiers au service des seconds.

Pour ce qui est de la Cour européenne des droits de l'homme, l'algorithme des chercheurs britanniques et américains pourrait l'aider à faire face à l'accumulation des dossiers à traiter et aux retards que cela entraîne — si bien qu'il lui faut parfois de longues années pour examiner des demandes visant justement à sanctionner certains États du fait de la lenteur de leurs justices nationales. L'algorithme, loin de décider à la place des magistrats, pourrait faciliter grandement leur travail en isolant les éléments pertinents pour le traitement des dossiers à l'aune des arrêts antérieurs, donnant ainsi des indices précieux quant aux issues prévisibles des procédures.

Pareille justice misant sur les nouvelles technologies de traitement de l'information est d'ores et déjà promue au Royaume-Uni, depuis qu'un rapport de 2015 a invité les pouvoirs publics à ouvrir le chantier d'une justice profitant des possibilités offertes par l'intelligence artificielle $^{1}$. Il s'agit moins d'un engouement technophile de la part du gouvernement britannique que d'une opportunité financière saisie : les frais de fonctionnement de la justice dématérialisée et en partie automatisée sont bien inférieurs à ceux de la justice physique et humaine.

Mais c'est avant tout aux États-Unis que la justice s'ouvre aux algorithmes — à tel point que l'expérience menée sur les arrêts de la Cour européenne des droits de l'homme n'y apparaît guère révolutionnaire. Dans beaucoup d'États américains, les procureurs recourent à des outils de justice prédictive afin de mesurer leurs chances de faire condamner certains suspects en fonction des preuves recueillies; et ils n'hésitent pas à abandonner les charges si l'issue des poursuites se révèle trop aléatoire. De plus, la détention provisoire et la libération conditionnelle sont désormais déterminées à l'aide d'un algorithme dénommé Compas ${ }^{2}$, lequel prend en compte une centaine de critères divers et variés. Cette aide informatique à la prise de décision - le détenu obtient un «score»sur une échelle allant de 1 à 10 , 1 correspondant à un risque de récidive très faible et 10 correspondant à un risque de récidive très élevé - a pour but de tout à la fois limiter les menaces de récidive et désengorger les prisons ${ }^{3}$. D'autres algorithmes sont de plus en plus fréquemment utilisés par les magistrats

\footnotetext{
${ }^{1}$ L'administration judiciaire britannique a annoncé la création en 2017 d'un tribunal civil en ligne pour les différends donnant lieu à des prétentions inférieures à 25000 livres. La première phase de la procédure consistera en des négociations assistées par ordinateur afin d'aboutir à une solution amiable. En cas de conflit persistant, le jugement sera rendu par internet.

2 Pour Correctional offender management profiling alternative sanctions.

${ }^{3}$ Si les résultats semblent prometteurs puisque les taux de récidive ont sensiblement diminué dans différents États, Compas a néanmoins été critiqué : quatre journalistes ayant comparé les parcours de 10000 détenus aux prévisions que l'algorithme avait réalisées les concernant ont constaté un biais ethnique : les noirs ont été systématiquement jugés plus susceptibles de récidiver, comme si l'un des critères les plus décisifs de l'algorithme résidait dans la couleur de peau (J. Larson, S. Mattu, L. Kirchner, J. Angwin, « How We Analyzed the COMPAS Recidivism Algorithm », Propublica 23 mai 2016 (disponible à l'adresse <www.propublica.org/article/how-we-analyzed-the-compas-recidivism-algorithm>)). En effet, 44,9\% des afroaméricains classés dans la catégorie des individus hautement susceptibles de récidiver n'ont dans les faits pas récidivé dans les deux années suivant leur libération, quand 23,5\% seulement des personnes blanches classées dans cette catégorie n'ont dans les faits pas récidivé. 47,7\% des personnes blanches jugées moyennement dangereuses ont récidivé, tandis que $28 \%$ des personnes noires jugées moyennement dangereuses ont récidivé. Les individus noirs ont donc deux fois plus de chances que les individus blancs d'être considérés à tort comme
} 
américains au moment de définir les peines de prison ou les éventuelles remises de peine. Et les résultats fournis par ces algorithmes sont désormais régulièrement cités afin de motiver les décisions.

En conséquence, peut-être la justice prédictive marche-t-elle avec le vent de l'histoire dans le dos et s'apprête-t-elle à conquérir l'Europe après avoir fait ses preuves outreAtlantique. À moins que, dans certains pays dont les cultures judiciaires diffèrent assez sensiblement de celle des États-Unis, et notamment en France, de fortes résistances ne l'empêchent. Par exemple, ne faudrait-il pas redouter le «panurgisme judiciaire » qui risquerait d'accompagner le déploiement de la justice assistée par ordinateur ? Les juges, sachant dans quel sens les tribunaux ont précédemment tranché les situations similaires, pourraient préférer suivre la tendance juridictionnelle afin de ne pas renvoyer l'image d'une justice balbutiante. Les algorithmes possèderaient ainsi un effet performatif, génèreraient des prophéties auto-réalisatrices. Or, si l'allégorie de la justice a les yeux bandés, n'est-ce pas afin de se prémunir contre l'influence des bases de données? Au-delà, certaines habitudes profondément ancrées et certaines exigences procédurales pourraient ralentir fortement l'avènement de la «justice 2.0 ». En définitive, peut-être l'avenir des outils de justice algorithmique, prédictive, prévisible, quantitative, statistique ou simulative appartient-il davantage au monde des avocats qu'à celui des magistrats.

\section{B. Possibilité et opportunité de l'algorithmisation du travail des avocats}

Les auteurs de l'expérience de déduction automatique des décisions de la Cour européenne des droits de l'homme espèrent que l'outil qu'ils ont créé connaîtra des applications pratiques, au-delà de son intérêt du point de vue scientifique - et théorique - : « Nous croyons que la construction d'un système prédictif basé sur les textes des décisions judiciaires peut offrir aux avocats un outil utile ${ }^{1}$. Plus qu'aux magistrats, c'est peut-être aux avocats que la justice prédictive s'adresse et s'adressera. Elle pourrait simplifier nombre de leurs tâches, leur permettre de gagner un temps précieux en les dispensant d'effectuer certaines recherches, donc leur permettre de se concentrer, par exemple, sur la préparation des plaidoiries ou sur les relations humaines avec les clients. Consulter la jurisprudence et effectuer des rapprochements avec les cas similaires déjà jugés a toujours constitué une partie importante du travail des avocats. Désormais, les algorithmes permettent d'accéder à certaines informations personnalisées, adaptées au profil et à la situation du justiciable, cela en quelques secondes quand, s'il fallait se plonger dans les recueils, cela prendrait plusieurs heures. Aussi, à l'heure du big data et de l'open data (que la récente loi «Pour une

potentiellement récidivistes. Et le Procureur général Eric Holder (l'équivalent du Ministre de la justice en France) de pointer du doigt les risques liés au recours aux algorithmes prédictifs : même s'ils sont conçus « avec les meilleures intentions », ils peuvent «exacerber des disparités injustes et injustifiées qui sont déjà trop communes dans notre système judiciaire et notre société » (cité par A. FRADIN, «États-Unis : un algorithme qui prédit les récidives lèse les Noirs », Rue 8924 mai 2016 (disponible à l'adresse <rue89.nouvelobs.com/2016/05/24/etats-unis-algorithme-predit-les-recidives-lese-les-noirs-264121>)).

${ }^{1}$ N. Aletras, V. LAmpos, D. Tsarapatsanis, D. PreoțiUC-Pietro, art. préc. 
République numérique » favorise ${ }^{1}$ ), serait-il peut-être difficilement justifiable de ne pas s'ouvrir à ces nouvelles technologies au nom de quelque orthodoxie, par exemple au nom de la « culture du papier $»^{2}$.

La justice prédictive, appelée à devenir de plus en plus pertinente à mesure que les algorithmes autoapprenants se perfectionneront et que le nombre d'informations leur permettant de s'améliorer grandira, peut constituer une aide précieuse à la prise de décision, servir à mieux conseiller et orienter les clients, à établir les meilleures stratégies contentieuses. Alors que l'appréciation ou quantification du risque est au cœur du métier d'avocat, les algorithmes tels que celui inventé par les scientifiques anglo-saxons doivent permettre d'estimer plus finement et plus efficacement ce risque ${ }^{3}$. Par conséquent, les cabinets d'avocats misant sur pareille intelligence artificielle disposeraient d'un avantage concurrentiel important. Et les algorithmes seraient à l'origine de réductions des coûts de traitement des dossiers, donc de gains de productivité et de compétitivité.

Aux États-Unis, l'algorithme Ross, développé par IBM, est déjà utilisé par de nombreux cabinets d'avocats. Quant à la société Lex Machina, elle a créé un algorithme qui pourrait devenir incontournable dans le secteur des brevets industriels. En France, ce genre d'outils émerge seulement. Des «legal start-up», notamment Case Law Analytics ${ }^{4}$ et Predictice ${ }^{5}$, ont élaboré des algorithmes permettant d'anticiper les issues des éventuelles actions en justice. Les procédures juridictionnelles ne sont que parfois bénéfiques mais sont toujours longues et coûteuses. Ces instruments peuvent aider à déterminer s'il serait judicieux ou vain de porter son litige devant les tribunaux, s'il serait préférable ou non de chercher un accord amiable.

Par suite, l'essor de ces technologies de justice prédictive, en entraînant une diminution du nombre d'actions en justice inopportunes, permettrait de réduire l'encombrement des tribunaux. Et cela ne serait pas sans conséquences sur l'exercice du métier d'avocat. Par exemple, en sachant que les chances pour son client d'obtenir gain de cause ne sont que de $5 \%$, le conseiller devrait tout mettre en œuvre afin d'éviter le jugement et recourir à un mode

${ }^{1}$ L. n ${ }^{\circ}$ 2016-1321, 7 oct. 2016, Pour une République numérique. Désormais, l'article L. 111-13 du Code de l'organisation judiciaire dispose que «les décisions rendues par les juridictions judiciaires sont mises à la disposition du public à titre gratuit dans le respect de la vie privée des personnes concernées ». Or les algorithmes de justice prédictive se nourrissent principalement des milliers de décisions de justice rendues au cours des dernières années — et qui sont désormais librement accessibles.

${ }^{2}$ En France, seul un avocat sur trois possède son propre site internet, ce qui interpelle tant le web est désormais le premier moyen de communication (L. NEUER, « Avocat : un métier en pleine mutation », [en ligne] <lepoint.fr>, 7 févr. 2017).

${ }^{3}$ Le fait que les algorithmes de justice prédictive interrogent le rapport au risque permet d'expliquer pourquoi ce sont principalement des compagnies d'assurance qui investissent dans la recherche et le développement de telles technologies.

${ }^{4}$ Case Law Analytics (<caselawanalytics.com>) quantifie l'aléa judiciaire, anticipe les décisions de justice et détermine le montant en euros que peut espérer toucher un justiciable dans différents types de litiges.

${ }^{5}$ Predictice (<predictice.com>), service lancé en septembre 2016, offre aux cabinets d'avocats et aux services juridiques des entreprises un outil capable d'estimer les chances d'obtenir gain de cause en cas de procédure judiciaire, cela dans toutes les branches du droit, du droit du travail au droit fiscal en passant par le droit des affaires et le droit administratif. Predictice permet également d'optimiser les stratégies contentieuses en identifiant et hiérarchisant les éléments pouvant influencer positivement l'issue du litige, les arguments pouvant le plus fortement toucher les juges et donc sur lesquels il serait opportun de se focaliser. Enfin, l'algorithme peut fournir une estimation des indemnités obtenues dans le cadre de contentieux similaires préalablement jugés et peut produire une carte des juridictions les plus favorables selon le domaine en cause. 
alternatif de résolution des conflits. La médiation et la transaction se développeraient à mesure que les prétoires se désengorgeraient. L'avocat devrait alors apprendre à jouer un rôle quelque peu différent.

Si les algorithmes venaient à prospérer et à conquérir les habitudes des avocats, ce ne serait rien de moins que la question d'une semi-robotisation de la défense et du conseil juridique qui se poserait. Aux États-Unis, les cabinets d'avocats ayant « recruté » l'algorithme Ross ont été amenés à se séparer de dizaines de collaborateurs jugés inutiles dès lors que Ross accomplit leurs tâches beaucoup plus rapidement et tout aussi bien qu'eux. Aussi une importante problématique que les «legaltechs» posent est-elle celle de la substitution de l'intelligence artificielle à l'intelligence humaine, du remplacement des collaborateurs humains par des collaborateurs électroniques, donc celle des suppressions d'emplois.

Par ailleurs, si les entreprises du secteur de la justice prédictive s'adressent pour l'heure essentiellement aux avocats, rien n'indique qu'elles ne chercheront pas demain à viser un public plus large ou que de nouveaux acteurs ne proposeront pas leurs services directement aux justiciables, lesquels en viendraient à se passer de plus en plus fréquemment des conseils des avocats. Les algorithmiques, allant jusqu'à fournir «clés en main » les arguments à présenter devant les juges, posent la question d'une future «ubérisation » de certains métiers de la justice ${ }^{1}$.

Néanmoins, plutôt que de mettre en péril les cabinets d'avocats, peut-être les services de justice algorithmique qui émergent pourraient-ils consolider leur caractère incontournable en les outillant mieux et en accentuant leur valeur ajoutée. Pour cela, il faudrait que la maîtrise de ces services demeure le propre des avocats - ce qui pourrait justifier une intervention règlementaire visant à encadrer le développement du marché de la justice prédictive. Les informations fournies par les algorithmes ne sauraient être acceptées sans contrôle, sans analyse, sans réflexion quant à leur pertinence, leurs conséquences et leurs implications juridiques. C'est pourquoi des juristes, des spécialistes des questions de droit et des rouages judiciaires capables, pour reprendre les termes ironiques de Balzac, de «légitimer les prétentions les plus absurdes $»^{2}$ et, surtout, de défendre les prétentions les plus légitimes en maniant l'argumentation et la rhétorique et en exploitant les zones grises de la loi, devraient toujours se trouver entre ces algorithmes et les justiciables.

\footnotetext{
${ }^{1}$ Déjà d'autres services font redouter pareille ubérisation. Tel est le cas, par exemple, d'eJust, plateforme d'arbitrage et de résolution en ligne des litiges commerciaux permettant de subroger dans différents cas des modes alternatifs de résolution des litiges à la justice ordinaire. Bien sûr, les avocats jouissent d'un monopole légal en matière de conseil juridique à titre principal ; et ils pourraient légitimement voir dans les «legaltechs » leur faisant concurrence des «braconniers du droit». Toutefois, le monopole attribué aux avocats se rapporte à des prestations de conseil juridique protéiformes s'inscrivant dans divers marchés. Or ces marchés ne sont, eux, soumis à aucun monopole légal. Certes, les services proposés par les avocats et par les legal start-up peuvent sembler substituables. Mais, lorsqu'ils délivrent une prestation de conseil juridique à titre principal, les avocats l'accompagnent généralement d'autres services pour lesquels ils ne disposent d'aucun monopole et qui peuvent donc être proposés par des concurrents non-avocats. Le droit exclusif relatif au conseil juridique à titre principal ne fait ainsi pas obstacle à l'existence de marchés où les avocats peuvent être concurrencés par les legal start-up. Par exemple, les tribunaux ont plusieurs fois confirmé la légalité du site <demanderjustice.com>, qui aide les justiciables à saisir la justice en quelques clics et à un faible coût, malgré les recours intentés par le Conseil national des barreaux et l'Ordre des avocats de Paris qui lui reprochent d'exercer illégalement la profession d'avocat (en dernier lieu, TGI Paris, 11 janv. 2017).

${ }^{2}$ H. DE BAlZAC, « Madame Firmiani », in Euvres complètes, t. I, Furne et Cie, 1846, p. 249.
} 
L'expérience des scientifiques britanniques et américains, ayant abouti à la création d'un véritable juge-robot en mesure de «dire le droit » ou, du moins, de «prédire le droit », est révélatrice des potentiels apports des algorithmes à l'objectif de valeur constitutionnelle de bonne administration de la justice ${ }^{1}$. Les «legaltechs» en général et les algorithmes en particulier pourraient, à l'avenir, se retrouver au cœur des rouages judiciaires, au cœur des pratiques et des habitudes des avocats et des magistrats. Mais cette expérience est également révélatrice des limites consubstantielles à ces nouveaux instruments et des dérives qui risqueraient d'accompagner leur mise en œuvre sans précautions.

Les algorithmes rassurent par leur logique mathématique implacable. Mais la justice peut-elle raisonnablement répondre à une logique mathématique implacable ? Des jugements rendus par ordinateur seraient-ils acceptables et acceptés ? Au-delà des menaces liées à une éventuelle dégradation de la qualité de la justice, peut-être sont-ce plus encore des considérations symboliques et psychologiques qui pourraient s'opposer à toute automatisation de la justice. Cette dernière s'appuie sur des cadres symboliques et sur une solennité qui s'effaceraient nécessairement à mesure qu'on recourrait aux algorithmes. L'humanité de la justice semble essentielle afin de lui permettre de conserver son aura et son prestige, donc son autorité. Ce sont par conséquent des limites symboliques et psychologiques plus que des limites technologiques qui pourraient freiner ou même interdire la grande transformation électronique de la justice.

En outre, les magistrats, au moment de juger, se fondent sur la loi et la jurisprudence, mais aussi sur l'éthique et autres formes de droit non officiel qui permettent d'adapter les décisions aux particularités de chaque cas. En témoigne l'exemple des « délits altruistes », ces infractions commises dans l'intérêt général et non afin d'en retirer un profit personnel ${ }^{2}$. Parfois, il est bon que la morale, forme de droit souple, tempère le droit dur. Or il est beaucoup plus délicat d'apprendre à des algorithmes le droit souple que le droit dur. C'est pourquoi le juge-humain ne saurait laisser sa place à un juge-robot. Et, pour les mêmes raisons, l'avocat-humain ne saurait s'effacer au profit d'un avocat-robot. Il faut gager que l'enjeu est et sera seulement celui du développement des aides algorithmiques permettant l'avènement d'un « magistrat augmenté » et d'un « avocat augmenté ».

La justice serait trop humaine pour que l'on puisse la confier à des robots ; et les robots seraient trop intelligents pour que l'on puisse se passer d'eux. À l'égard de cette problématique comme souvent, tout ne serait que question d'équilibre: équilibre entre technophobie et technophilie afin que la justice algorithmique exprime tout son potentiel sans

\footnotetext{
${ }^{1}$ Cons. const., déc. $n^{\circ} 2009-595$ DC, 3 déc. 2009, Loi organique relative à l'application de l'article 61-1 de la Constitution, considérant 4.

${ }^{2}$ La situation des lanceurs d'alerte dans l'affaire dite «Luxleaks » est symptomatique. Ceux-ci, que ce soit en première instance ou bien en appel, ont certes été condamnés puisqu'ils avaient commis des infractions et non des moindres («vol et violation du secret professionnel et du secret des affaires », ainsi que «fraude informatique, blanchiment et divulgation du secret des affaires »), mais des peines très clémentes leur ont été infligées. La justice luxembourgeoise a en effet pris en compte le caractère d'intérêt général de leurs révélations.
} 
déstabiliser les professions d'avocat et de magistrat ni nuire aux intérêts des justiciables. Les algorithmes qui gagnent progressivement le paysage du droit et de la justice se présentent à la fois telle une opportunité à saisir et tel un danger à prévenir. Ils pourraient aussi bien susciter un renouveau et une modernisation des métiers d'avocat et de magistrat que provoquer leur affaiblissement en les banalisant ou en les gadgétisant par trop. Tout dépendra, d'une part, des niveaux d'utilité et de fiabilité qu'ils atteindront et, d'autre part, de l'accueil que les juges et les avocats leur réserveront. 